\title{
Functionalized 2PP structures for the BioPhotonics Workstation
}

Matsuoka, Tomoyo; Nishi, Masayuki; Sakakura, Masaaki; Miura, Kiyotaka; Hirao, Kazuyuki; Palima, Darwin; Tauro, Sandeep; Bañas, Andrew Rafael; Glückstad, Jesper

\section{Published in:}

Proceedings of SPIE - The International Society for Optical Engineering

Link to article, DOI:

10.1117/12.877189

Publication date:

2011

Document Version

Publisher's PDF, also known as Version of record

Link back to DTU Orbit

Citation (APA):

Matsuoka, T., Nishi, M., Sakakura, M., Miura, K., Hirao, K., Palima, D., Tauro, S., Bañas, A. R., \& Glückstad, J. (2011). Functionalized 2PP structures for the BioPhotonics Workstation. Proceedings of SPIE - The International Society for Optical Engineering, 7950, 79500Q. https://doi.org/10.1117/12.877189

\section{General rights}

Copyright and moral rights for the publications made accessible in the public portal are retained by the authors and/or other copyright owners and it is a condition of accessing publications that users recognise and abide by the legal requirements associated with these rights.

- Users may download and print one copy of any publication from the public portal for the purpose of private study or research.

- You may not further distribute the material or use it for any profit-making activity or commercial gain

- You may freely distribute the URL identifying the publication in the public portal 


\title{
Functionalized 2PP structures for the BioPhotonics Workstation
}

\author{
Tomoyo Matsuoka*a, Masayuki Nishi ${ }^{\mathrm{a}}$, Masaaki Sakakura ${ }^{\mathrm{a}}$, Kiyotaka Miura ${ }^{\mathrm{a}}$, Kazuyuki Hirao $^{\mathrm{a}}$, \\ Darwin Palima ${ }^{\mathrm{b}}$, Sandeep Tauro ${ }^{\mathrm{b}}$, Andrew Bañas ${ }^{\mathrm{b}}$ and Jesper Glückstad ${ }^{\mathrm{b}}$ \\ ${ }^{a}$ Dept. of Material Chemistry, Graduate School of Engineering, Kyoto University, Nishikyo-ku, \\ Kyoto 615-8510, Japan \\ ${ }^{b}$ DTU Fotonik, Dept. of Photonics Engineering, Technical University of Denmark, Ørsted Plads 343, \\ DK-2800 Kgs. Lyngby, Denmark
}

\begin{abstract}
In its standard version, our BioPhotonics Workstation (BWS) can generate multiple controllable counter-propagating beams to create real-time user-programmable optical traps for stable three-dimensional control and manipulation of a plurality of particles. The combination of the platform with microstructures fabricated by two-photon polymerization (2PP) can lead to completely new methods to communicate with micro- and nano-sized objects in 3D and potentially open enormous possibilities in nano-biophotonics applications. In this work, we demonstrate that the structures can be used as microsensors on the BWS platform by functionalizing them with silica-based sol-gel materials inside which dyes can be entrapped.
\end{abstract}

Keywords: Optical manipulation, Generalized phase contrast, two-photon polymerization, optical sensor, sol-gel coating

\section{INTRODUCTION}

Optical trapping was first reported by Ashkin ${ }^{1}$ in 1970 and has been attracting more and more attention these days especially in biological fields because it enables us to manipulate microscopic objects three-dimensionally in real-time without any mechanical contact with them ${ }^{2}$. The technique generally uses the forces of radiation pressure arising from the momentum of light itself. Among many systems currently developed for optical trapping, our BioPhotonics Workstation (BWS) has some advantages over other systems; it can generate multiple controllable counter-propagating beams to create real-time user-programmable optical traps for stable three-dimensional control and manipulation of a plurality of particles. We have traditionally used the counter-propagating geometry to achieve stable three-dimensional trapping while maintaining a large working distance by using microscope objectives with relatively low numerical apertures. The BWS affords independent control of the counter-propagating beam patterns and we have earlier exploited this to correctly match the corresponding set of counter-propagating beam traps ${ }^{3}$. With this trapping geometry we are currently able to generate around 100 dynamic optical traps using well-separated objectives, which eliminates the need for high numerical aperture oil or water immersion objectives required in conventional optical tweezers. This generates a large field of view and leaves vital space for integrating other enabling tools for probing or processing the trapped particles, such as linear and nonlinear microscopy or pulsed laser processing.

Optical trapping is usually demonstrated with dielectric spheres and biological samples. But trapping applications extend beyond readily available samples such as custom designed two-photon polymerized (2PP) structures. Two-photon polymerization has emerged as a promising technique for fabrication of three-dimensional structures ${ }^{4}$. In the $2 \mathrm{PP}$ process, femtosecond laser pulses induce nonlinear absorption in a highly localized focal volume which leads to threedimensional micro- or nanoscopic structures. Not only does it enable flexible fabrication of polymer structures, but it also can be applied for fabrication of new micro/nanostructures or advanced photo-excitations which have functions for communicating with other materials ${ }^{5}$. By combining the functionalized 2PP structures with the BWS platform, novel methods for analyzing and/or processing objects can be realized independently and fully decoupled from the counterpropagating trapping geometry.

Considering the potential of the BWS platform in biological applications ${ }^{6,7}$, we were motivated to fabricate $2 \mathrm{PP}$ structures functionalized with fluorescent dyes which can act as sensors for specific ions or temperature in biological environments. This kind of optical sensor can be designed by applying conventional techniques for fiber-optic sensors

Complex Light and Optical Forces V, edited by David L. Andrews, Enrique J. Galvez, Jesper Glückstad, Proc. of SPIE Vol. 7950, 79500Q · C 2011 SPIE · CCC code: 0277-786X/11/\$18 - doi: 10.1117/12.877189 
(optodes) ${ }^{8,9}$. While using optodes is basically an indirect sensing method and the fiber connection to external devices sometimes prevents flexible approach to analytes, 2PP structures are expected to work more flexibly and make it possible to detect environmental changes directly by monitoring the fluorescence change of the dyes on a trapping system. Since the surfaces of the structures obtained using normal epoxy- or acryl-based resins are chemically inert in general, functionalization of them directly with dye molecules is not straightforward. Therefore, we decided to first introduce hydroxyl and/or methoxy groups in the structures by adding a silane coupling agent with epoxy group to a commercially-available epoxy-based resin. These two materials can be copolymerized through ring-opening polymerization process of epoxy groups, followed by developing and washing processes, possibly leading to the structures with hydroxyl and/or methoxy groups on the surface. The next step is to coat the obtained structures with silica-based sol-gel materials which entrap dyes inside them. The hydrolysis and polycondensation reactions between the surface and silane coupling agent in a coating solution result in covalent bond formation (-Si-O-Si-) at the interface between the structures and the coating materials. The highly porous nature of sol-gel materials makes them excellent hosts for sensing molecules, since the species to be sensed can easily diffuse towards the sensing center while the matrix improves biocompatibility by protecting the biological environment from any potentially toxic effects of the sensing dye ${ }^{10}$.

One issue when fabricating microscopic objects using $2 \mathrm{PP}$ by a single laser beam is that it needs long processing times. The long processing time makes practical application of $2 \mathrm{PP}$ difficult. To solve this problem, we utilized a phase modulation technique with a spatial light modulator (SLM) ${ }^{11}$. The technique allows us to split one laser beam into multiple beams by means of computer generated holograms (CGH). Multiple focused beam spots can be created after focusing, depending on the spatial phase modulation. At each beam focus, the resin is photopolymerized through twophoton absorption, which leads to the creation of polymerized materials at multiple positions at the same time. By combining CGH with computer-generated Fresnel lenses, a flexible and fast two-photon three-dimensional (3D) microfabrication can be realized.

\section{EXPERIMENTAL METHODS}

\subsection{PP processing}

The experimental setup for 2PP with an SLM is shown in Fig. 1. Amplified Ti: $\mathrm{Al}_{2} \mathrm{O}_{3}$ femtosecond laser pulses (MiraLegend, Coherent Inc.) with a pulse duration of about $120 \mathrm{fs}$, central wavelength of $800 \mathrm{~nm}$ and repetition rate of $1 \mathrm{kHz}$ were used for the process. The laser pulses were reflected on a liquid crystal on silicon SLM (LCOS-SLM, X10468-02, Hamamatsu Photonics K.K.), which had $800 \times 600$ square pixels with a pixel pitch of $20 \mu \mathrm{m}$ and that can provide a reflected beam with an independent phase change of up to $2 \pi$ at each pixel ${ }^{12}$. The laser pulses reflecting on the SLM then passed through a telescope, which consisted of two concave lenses (magnification of $\mathrm{M}=0.3$ ), and were focused inside a pre-baked resin with an oil immersion objective lens (NA = 1.40; Plan Apo VC, Nikon). The resin was prepared by mixing SU-8 3035 (NIPPON KAYAKU) and 3-glycidyloxypropyltrimethoxysilane (Aldrich) in 2:1 volume ratio. The mixture was then spin-coated on a cover glass substrate and pre-baked at $95{ }^{\circ} \mathrm{C}$ for 1 hour. The substrate was placed on a 3D translation stage and the spatially phase modulated beam was focused at multiple positions inside the resin. The processing of the structures was observed with a CCD camera and back illumination with a red LED lamp, although the resin shows only a slight change after laser irradiations because the refractive index change of SU-8 occurs not in the irradiation process but in the post-baking process. The laser beam power was controlled at $30 \mathrm{~mW}$ with a neutral density (ND) filter, and the exposure time for each hologram was controlled at $30 \mathrm{~ms}$ with a mechanical shutter. The translation stage, LCOS-SLM and mechanical shutter were controlled by a personal computer. The CGHs were calculated by a simple iterative-Fourier-transform algorithm (IFTA) in which the optimization are made by setting a non-zero amplitude to the dark pixels as well as adding a dummy area outside the signal area. A calculated CGH with $512 \times 512$ pixels was displayed in the central region of the active area of the LCOS-SLM. The 3D processing was performed by simply superimposing phase Fresnel lens onto the $\mathrm{CGH}$, which leads to a focus shift of multiple spots generated by each hologram. By switching these combined CGHs layer by layer, fast 3D processing was achieved.

It should be noted that for the structure to be released from the substrate after developing but not to be washed away, the 3D writing should start from an inside point of the resin and end at an interface point between the glass and the resin. 


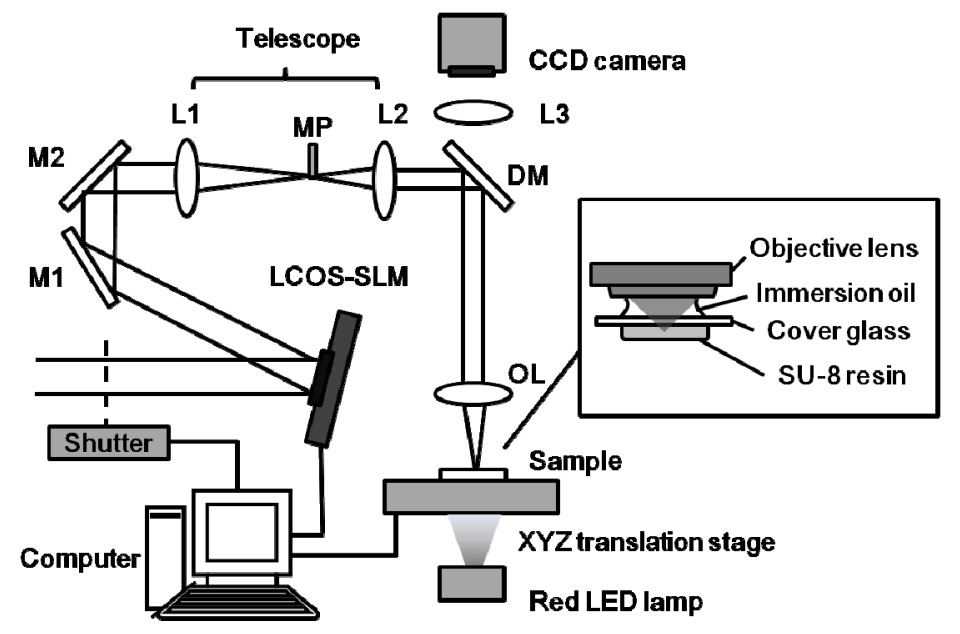

Figure 1. Experimental setup for 2PP fabrication with an SLM. M1, M2: dielectric mirrors; $\mathrm{L} 1$ : a lens of $\mathrm{f}=500 \mathrm{~mm}$; $\mathrm{L} 2$ : a lens of $\mathrm{f}=150 \mathrm{~mm}$; DM: a dichroic mirror which reflects light of $750-850 \mathrm{~nm}$; OL: an objective lens; MP: a metal plate.

\subsection{Functionalization}

The laser-processed resin was first post-baked at $95{ }^{\circ} \mathrm{C}$ for 15 minutes, followed by developing for 15 minutes in a SU-8 developer (NIPPON KAYAKU) and rinsing with isopropanol. To activate the surface silanol groups of the fabricated structure, the substrate was then dipped in $0.1 \mathrm{M}$ aqueous solution of hydrochloric acid for 10 seconds and dried in air. Preparation of sol-gel solution for coating was performed according to the work reported in literature ${ }^{13}$. A prehydrolysed solution was prepared by heating an ethanolic solution containing tetramethoxysilane (TMOS), water and hydrochloric acid in the following molar ratio: 1 TMOS: $3 \mathrm{EtOH}: 5 \times 10^{-5} \mathrm{HCl}: 1 \mathrm{H}_{2} \mathrm{O}$ at $100{ }^{\circ} \mathrm{C}$ for $1 \mathrm{~h}$. Then, cetyltrimethylammonium bromide (CTAB) was dissolved in ethanol and added to the prehydrolysed solution together with an additional amount of water and $\mathrm{HCl}$. The final molar ratio was 1 TMOS: $20 \mathrm{EtOH}: 0.004 \mathrm{HCL}: 5 \mathrm{H}_{2} \mathrm{O}: 0.10$ CTAB. The final solution was then stirred for four days at room temperature. $1 \mathrm{ml}$ of a dye ethanol solution $(0.13 \mathrm{mM})$ is then mixed with $1 \mathrm{ml}$ of the sol and left under stirring for several hours. A typical dye used in this work was Fluo-5N (Invitrogen). Coating of 2PP structures were performed by spin-coating the resulting solution on the glass substrate with the structures and then the coated structures were gently released from the substrate by a glass needle and dried in air at room temperature. We also spin-coated the sol on a clean cover glass to investigate the response of the sol-gel film. Before use, the coated structures and films were placed in pure water to remove excess and unbound dyes.

\subsection{Analysis}

An FE-SEM (JSM6700F, JEOL) and a Raman microspectrometer (Nanofinder30, Tokyo Instruments; wavelength=633 $\mathrm{nm}, \mathrm{NA}=0.6$ ) was used to investigate and analyze the $2 \mathrm{PP}$ fabricated structures. The response of the sol-gel film coated on a cover glass was measured using a spectrofluorometer (Fluoromax-P, Jobin Yvon Horiba). The response of the solgel coated structures was observed using a fluorescence microscope with excitation at $490 \mathrm{~nm}$. These fluorescence measurements were performed by first immersing the structures or the film into solutions with various concentrations of calcium ions for 10 minutes and then drying them in air for a few minutes.

\section{RESULTS AND DISCUSSIONS}

Since we used a resin prepared by mixing SU-8 and 3-glycidyloxypropyltrimethoxysilane in 2:1 volume ratio, the surface chemical property of the fabricated structures are supposed to be modified as illustrated in Figure 2. The fabricated structures are expected to have hydroxyl and/or methoxy groups derived from 3glycidyloxypropyltrimethoxysilane, which play important roles in forming covalent bonds between the surface of the 
polymer and a sol-gel coating. We confirmed this by the observed difference between the coating strengths; the sol-gel coating on SU-8 film was easily peeled off from a polymerized SU-8 surface upon immersion in water, while it stuck stably to the surface of a modified SU-8.

\section{Step 1: Copolymerization of SU-8 and 3-Glycidyloxypropyltrimethoxysilane}

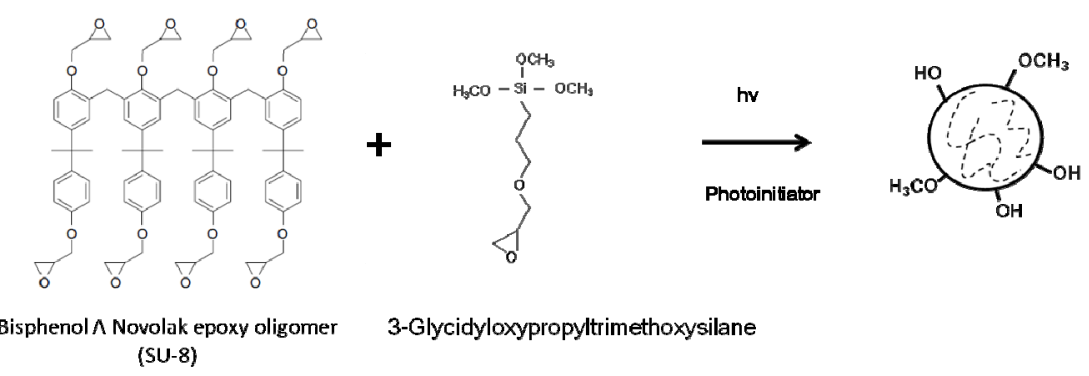

Step 2: Sol-gel coating of the copolymerized structures
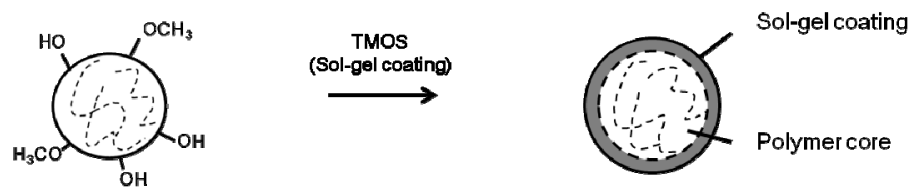

Figure 2. Schematic representation of chemical steps for functionalization

Figure 3 shows an SEM image of the fabricated structures. Since we used 18 holograms in total for each structure, laser processing for each structure was finished within $1 \mathrm{~s}$. Although the processing can be finished much faster than that of conventional scanning system, the fabricated structures are not smooth and sometimes randomly rough because of the inhomogeneous nature of laser spots created by the SLM and the constraint on spacing between spots imposed by the discrete Fourier transform procedure. However, considering long processing times of conventional 2PP systems, we believe that our multi-focus approach using LCOS-SLM is acceptable when the smoothness and fineness of the structures are not necessarily required. Introducing additional algorithms such as corrections to the optimal-rotationangle method would improve the uniformity of the spots ${ }^{14,15}$, and the spatial configuration of the focus spots could also be optimized (e.g., by using the method reported by Bengtsson et $\mathrm{al}^{16}$ ). The poor quality of the structures might also be due to the high repetition rate of the femtosecond laser, i.e., the high energy of single pulses, which possibly causes boiling or destroying of the resin. We need to investigate and improve these points further in future works.

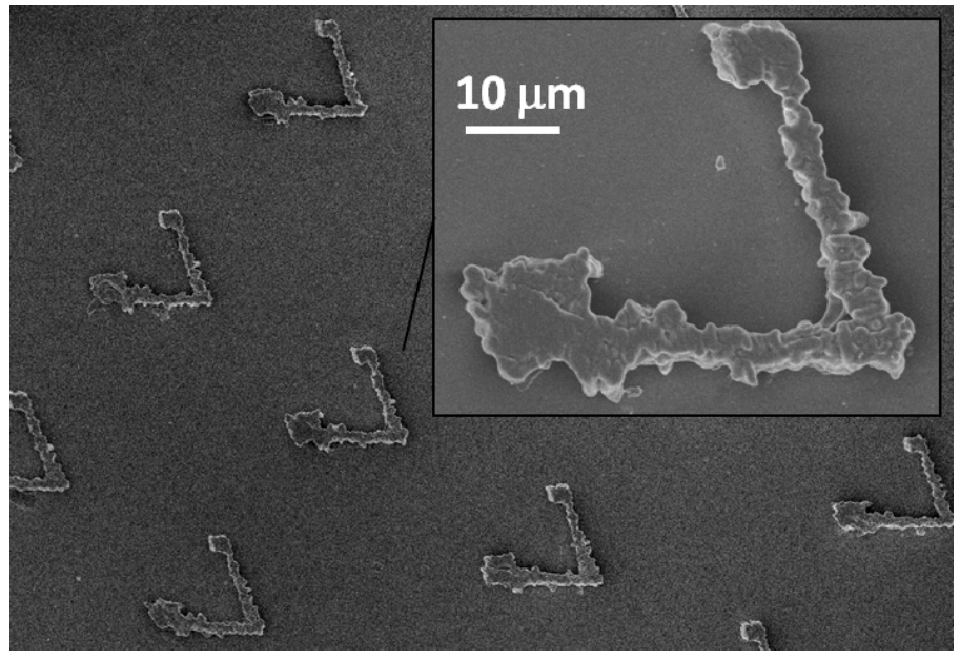

Figure 3. SEM images of the obtained 2PP structures 
Figure 4 shows the fluorescence emission spectra of the sol-gel film coated on a cover glass. It can be seen that the entrapped calcium indicator (Fluo-5N) in sol-gel matrix exhibits an emission band around $530 \mathrm{~nm}$ whose intensity depends on the concentration of calcium ion. It is clear that the entrapped dyes remain sensitive in the sol-gel matrix. We also confirmed that the response was reversible and reproducible even after several measurement cycles. Although there was also the leaching of dye molecules out of the matrix to some extent, we think this is not of much concern for short time or disposable use of the sensors.

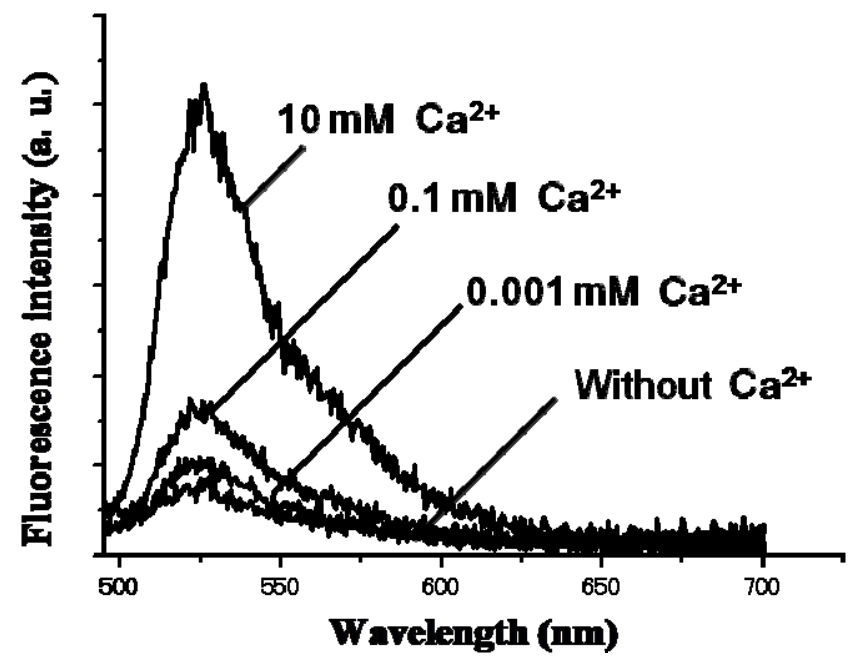

Figure 4. Fluorescence emission spectra of sol-gel film coated on cover glass.

Figure 5 shows fluorescence microscope images of a 2PP structure with sol-gel coating. These images were captured in the same optical condition before (a) and after (b) immersing it in a $10 \mathrm{mM}$ solution of calcium ions. We can clearly see the difference which arises from the response of the entrapped calcium indicators. The sol-gel matrix contained only one dye in this work, but multiple dyes can also be combined in a single matrix, so that a reference standard can be used to minimize possible errors. Additionally, we can potentially entrap any dye inside the matrix. Metal nanoparticles, quantum dots and even enzymes can also be entrapped. By introducing these structures on the BWS platform, a real-time and flexible measurement of biologically important ions and temperature would be realized.
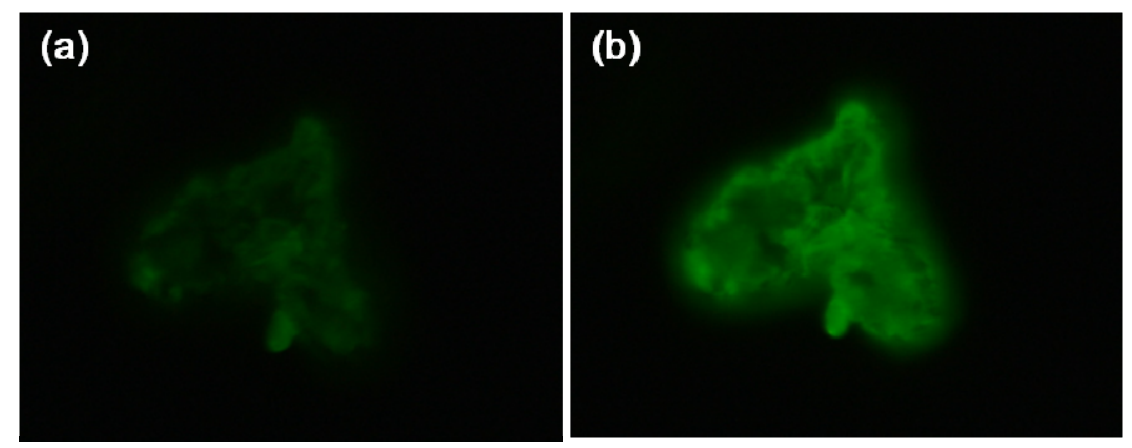

Figure 5. Fluorescence images with excitation at $490 \mathrm{~nm}$ : (a) sol-gel coated structure in water and (b) in $10 \mathrm{mM} \mathrm{Ca}^{2+}$ solution. The structure were immersed in the respective solution for 10 minutes and dried before measurements. 


\section{CONCLUSION}

We used 2PP processing and surface modification to fabricate functionalized microstructures which can act as sensors for calcium ion. Commercially-available SU-8 was modified by mixing with 3-glycidyloxypropyltrimethoxysilane in order to introduce hydroxyl and/or methoxy groups on the surface of the fabricated structures. To speed up the 2PP processing, we utilized phase modulation technique with a SLM. Although the processing can be finished much faster than that of conventional scanning system, it turns out that the fabricated structures are rough because of the inhomogeneous nature of laser spots created by the SLM and the constraint on spacing between spots imposed by the discrete Fourier transform procedure. Functionalization of the structures was performed by coating them with silicabased sol-gel materials which contain fluorescent calcium indicator inside. The fluorescence measurements showed that entrapped dyes remain sensitive in the sol-gel matrix, indicating the potential of the coated structures as sensors in biological environments. Future work will focus on real-time measurement of the change of biological environment using the fabricated structures as user-controlled sensors in the BioPhotonics Workstation. Entrapping various other materials inside the sol-gel coating should also be investigated.

\section{REFERENCES}

[1] Ashkin, A., "Acceleration and Trapping of Particles by Radiation Pressure," Phys. Rev. Lett. 24, 156-159 (1970)

[2] Glückstad, J., "Sorting particles with light," Nature Materials 3 (1), 9-10 (2004)

[3] Rodrigo, P. J., Kelemen, L., Palima, D., Alonzo, C. A., Ormos, P. and Glückstad, J., “Optical microassembly platform for constructing reconfigurable microenvironments for biomedical studies," Opt. Express 17(8), 6578 (2009)

[4] Kawata, S., Sun, H. B., Tanaka, T. and Takada, K., "Finer features for functional microdevices," Nature 412, 697-698 (2001)

[5] Papagiakoumou, E., Anselmi, F., Begue, A., de Sars, V., Glückstad, J., Isacoff, E. and Emiliani, V., "Scanless two-photon excitation of channelrhodopsin-2", Nature Methods 7, 848-854 (2010).

[6] Ulriksen, H. U., Thøgersen, J., Keiding, S., Nielsen, I. P., Dam, J., Palima, D. Z., Stapelfeldt, H. and Glückstad, J., "Independent trapping, manipulation and characterization by an all-optical biophotonics workstation", J. Europ. Opt. Soc. Rap. Public. 3, 08034 (2008).

[7] Glückstad, J., Rodrigo, P. J. and Nielsen, I. P., "Optical 3D Manipulation and Observation in Real-Time (Invited Paper)," Journal of Robotics and Mechatronics 18 (6), 692-697 (2006).

[8] Barker, S. L. R., Thorsrud, B. A. and Kopelman, R., "Nitrite- and Chloride-Selective Fluorescent NanoOptodes and in Vitro Application to Rat Conceptuses," Anal. Chem. 70, 100-104(1998)

[9] Morf, W. E., Seiler, K., Rusterholz, B. and Simon, W., "Design of a novel calcium-selective optode membrane based on neutral ionophores," Anal. Chem. 62 (7), 738-742(1990)

[10] MacCraith, B. D., McDonagh, C., Mcevoy, A. K., Butler, T., OKeeffe, G. and Murphy, V.,"Optical chemical sensors based on sol-gel materials: recent advances and critical issues," J. Sol-Gel Sci. Technol. 8, 10531061(1997)

[11] Sakakura, M., Sawano, T., Shimotsuma, Y., Miura, K. and Hirao, K., "Parallel Drawing of Multiple Bent Optical Waveguides Using a Spatial Light Modulator," Jpn. J. Appl. Phys. 48, 126507 (2009)

[12] Inoue, T., Tanaka, H., Fukuchi, N., Takumi, M., Matsumoto, N., Hara, T., Yoshida, N., Igasaki, Y. and Kobayashi, Y., "LCOS spatial light modulator controlled by 12-bit signals for optical phase-only modulation," Proc. SPIE Y11, 6487(2007)

[13] Miled, O. B., Grosso, D., Sanchez, C. and Livage, J., "An optical fibre pH sensor based on dye doped mesostructured silica" Journal of Physics and Chemistry of Solids 65(10), 1751-1755(2004)

[14] Bengtsson, J., "Kinoform design with an optimal-rotation-angle method,” Appl. Opt. 33(29), 6879-6884 (1994)

[15] Takahashi, H., Hasegawa, S. and Hayasaki, Y., "Holographic femtosecond laser processing using optimalrotation-angle method with compensation of spatial frequency response of liquid crystal spatial light modulator," Appl. Opt. 46, 5917-5923 (2007)

[16] Engström, D., Frank, A., Backsten, J., Goksör, M. and Bengtsson, J., "Grid-free 3D multiple spot generation with an efficient single-plane FFT-based algorithm," Opt. Express 17, 9989-10000 (2009) 